\title{
Potential Therapeutic Effects of Curcumin on Glycemic and Lipid Profile in Uncomplicated Type 2 Diabetes-A Meta-Analysis of Randomized Controlled Trial
}

\author{
Emma Altobelli ${ }^{1,2, *}$, Paolo Matteo Angeletti ${ }^{1,3}$, Ciro Marziliano ${ }^{1}$, Marianna Mastrodomenico ${ }^{1}$, \\ Anna Rita Giuliani ${ }^{1}$ and Reimondo Petrocelli ${ }^{4}$
}

1 Department of Life, Public Health and Environmental Sciences, University of L'Aquila, 67100 L'Aquila, Italy; paolomatteoangeletti@gmail.com (P.M.A.); c.marziliano@univaq.it (C.M.); mariannamastrodomenico@gmail.com (M.M.); annarita.giuliani@univaq.it (A.R.G.)

2 Epidemiology and Biostatistics Unit, Local Health Unit, 64100 Teramo, Italy

3 Rianimazione e TIPO Cardiochirurgica, Ospedale G. Mazzini, Local Health Unit, 64100 Teramo, Italy

4 Public Health Unit, ASREM, 86100 Campobasso, Italy; reimondo.petrocelli@asrem.org

* Correspondence: emma.altobelli@univaq.it; Tel.: +39-0862-434666; Fax: +39-0862-432903

check for updates

Citation: Altobelli, E.; Angeletti, P.M.; Marziliano, C.;

Mastrodomenico, M.; Giuliani, A.R.;

Petrocelli, R. Potential Therapeutic

Effects of Curcumin on Glycemic and Lipid Profile in Uncomplicated Type 2 Diabetes-A Meta-Analysis of Randomized Controlled Trial. Nutrients 2021, 13, 404. https:// doi.org/10.3390/nu13020404

Academic Editor: Roberta Masella

Received: 3 January 2021

Accepted: 25 January 2021

Published: 27 January 2021

Publisher's Note: MDPI stays neutral with regard to jurisdictional claims in published maps and institutional affiliations.

Copyright: (c) 2021 by the authors. Licensee MDPI, Basel, Switzerland. This article is an open access article distributed under the terms and conditions of the Creative Commons Attribution (CC BY) license (https:/ / creativecommons.org/licenses/by/ $4.0 /)$.

\begin{abstract}
Diabetes mellitus is an important issue for public health, and it is growing in the world. In recent years, there has been a growing research interest on efficacy evidence of the curcumin use in the regulation of glycemia and lipidaemia. The molecular structure of curcumins allows to intercept reactive oxygen species (ROI) that are particularly harmful in chronic inflammation and tumorigenesis models. The aim of our study performed a systematic review and meta-analysis to evaluate the effect of curcumin on glycemic and lipid profile in subjects with uncomplicated type 2 diabetes. The papers included in the meta-analysis were sought in the MEDLINE, EMBASE, Scopus, Clinicaltrials.gov, Web of Science, and Cochrane Library databases as of October 2020. The sizes were pooled across studies in order to obtain an overall effect size. A random effects model was used to account for different sources of variation among studies. Cohen's $d$, with $95 \%$ confidence interval (CI) was used as a measure of the effect size. Heterogeneity was assessed while using $Q$ statistics. The ANOVA-Q test was used to value the differences among groups. Publication bias was analyzed and represented by a funnel plot. Curcumin treatment does not show a statistically significant reduction between treated and untreated patients. On the other hand, glycosylated hemoglobin, homeostasis model assessment (HOMA), and low-density lipoprotein (LDL) showed a statistically significant reduction in subjects that were treated with curcumin, respectively $(p=0.008, p<0.001$, $p=0.021$ ). When considering HBA1c, the meta-regressions only showed statistical significance for gender $(p=0.034)$. Our meta-analysis seems to confirm the benefits on glucose metabolism, with results that appear to be more solid than those of lipid metabolism. However, further studies are needed in order to test the efficacy and safety of curcumin in uncomplicated type 2 diabetes.
\end{abstract}

Keywords: curcuma; turmeric; type 2 diabetes; dyslipidemia; meta-analysis; randomized control trial

\section{Introduction}

Type 2 diabetes (T2DM) is an important issue for public health, and it is growing in the world; in fact, according to the World Health Organization (WHO) report in 2016, 422-million people are diagnosed with diabetes [1]. In Europe, $50 \%$ of Countries show T2DM prevalence rates in the range of 8-9\% [2]. Bommer et al. have demonstrated that the global costs of T2DM and its consequences are large, and they will substantially increase by 2030 [3].

Lifestyle risk factors that are related to diabetes, as obesity and overweight are two major risk factors. The treatment of T2DM includes the use of anti-diabetic drugs and prevention based on lifestyle habits. In recent years, there has been growing research interest on the efficacy evidence of curcumin use in the regulation of glycemia and lipidaemia [4]. 
Curcumin is the main bioactive component that is extracted from the rhizome of Curcuma Longa. It is a product used since ancient times, in cuisine, as in traditional medicine [5]. The properties that are attributed to curcumin are remarkable: in fact, it has an antioxidant and anti-inflammatory effect [6].

Its molecular structure makes it possible to intercept reactive oxygen species (ROI) that are particularly harmful in chronic inflammation and tumorigenesis models [5].

Curcumin can have a therapeutic effect on some chronic diseases, such as rheumatoid arthritis, coronary artery disease, atherosclerosis, T2DM, and obesity [7].

Ramirez-Bosca et al. showed that daily treatment with curcumin can decrease the low-density lipoprotein (LDL) in healthy subjects [8]. In addition, Mohammadi et al. [9] demonstrated that one-month oral administration of curcumin (1 gram/day) could reduce the triglycerides concentrations in obese subjects. Rahimi et al., in a randomized trial, highlighted that curcumin reduces HbA1c during three months of therapy [10].

In order to evaluate its effectiveness, some studies have been conducted on the effects of curcumin on glycemic and lipid control in subjects with uncomplicated T2DM [10-16].

In this systematic review and meta-analysis, we aim to evaluate the effect of curcumin on glycemic and lipid profile in subjects with uncomplicated T2DM.

\section{Materials and Methods}

The papers that were included in the meta-analysis were sought in the MEDLINE, EMBASE, Scopus, Clinicaltrials.gov, Web of Science, and Cochrane Library databases as of October 2020. The search terms used were: curcuma or curcumins or turmeric AND type 2 diabetes or diabetes; ("Diabetes Mellitus, Type 2" [Mesh]) AND ("Curcuma" [Mesh]) and applied the following filters: humans, published articles from 2000 to 2020, and clinical trials.

The papers were selected while using the Preferred Reporting Items for Systematic Reviews and Meta-Analyses (PRISMA) flowchart (Figure 1) and the PRISMA checklist (Table S1) [17]. A manual search of possible references of interest was also performed. Only studies that were published in English were considered. The papers were selected by two independent reviewers (P.M.A. and C.M); a methodologist (E.A.) resolved any disagreements. Bias was assessed using the Cochrane Collaboration tool for assessing the risk of bias (Table S2) [18].
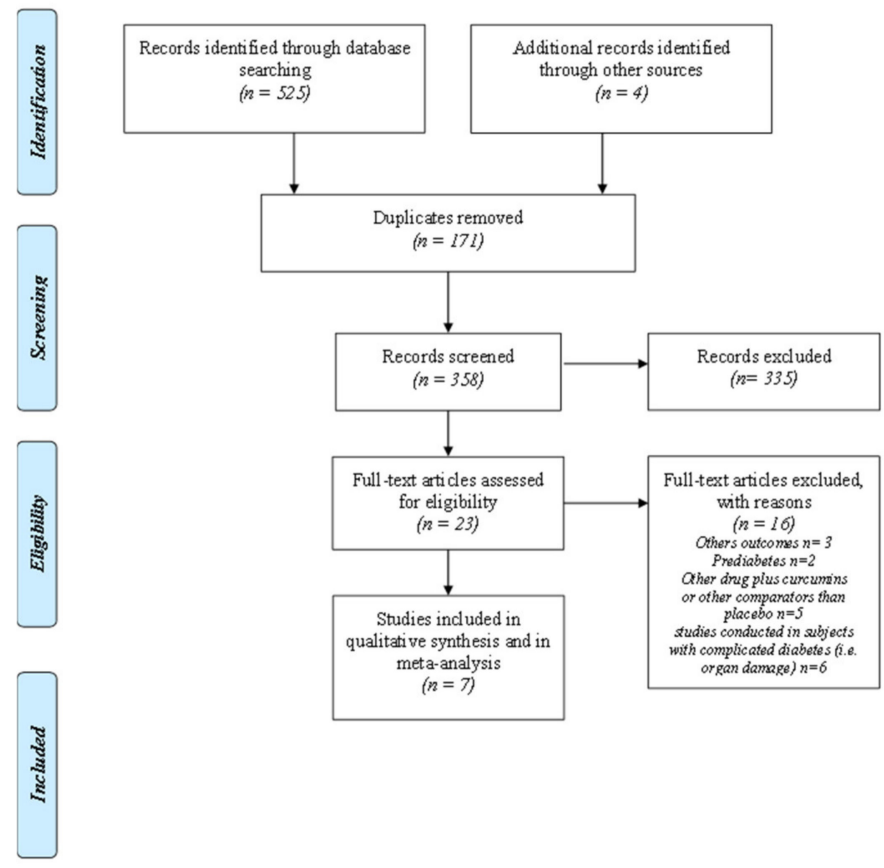

Figure 1. Prisma Flow-chart. 


\section{Statistical Analysis}

The sizes were pooled across studies in order to obtain an overall effect size. A random effects model was used to account for the different sources of variation among studies [19]. Cohen's d, with $95 \%$ confidence interval (CI) and $p$-value, was used as a measure of effect size [20]. Heterogeneity was assessed using $Q$ statistics, $I^{2}$, Tau, and Tau2. The stability of study findings was checked with moderator analysis. A subgroups analysis was also performed while considering the country of primary studies, because four of seven studies were conducted in Iran. The ANOVA-Q test was used to value the differences among groups. Publication bias was analyzed and represented by a funnel plot; funnel plot symmetry was assessed with Egger's test [21]. Finally, publication bias was checked using the trim and fill procedure; we used Rosenthal's estimator and the fail-safe number to analyze publication bias [22]. Finally, meta-regression analyses were utilized for the following variables: article publication year, gender, age, and dose. Regression models were applied for continuous variables. Meta-regressions were performed when the number of studies containing the variables to be analyzed was $\geq 4$. PROMETA 3 software (IDo Statistics-Internovi, Cesena, Italy) was used. The considered outcomes were body mass index (BMI), homeostasis model assessment-insulin resistance index (HOMA-IR), glycosylated hemoglobin (Hb1Ac), Triglycerides (TG), Total Cholesterol (TC), High-density lipoprotein (HDL), and LDL. All of the values were reported in $\mathrm{mg} / \mathrm{mL}$ while using a conversion formula [23].

\section{Results}

The literature search highlighted the presence of 529 references (Figure 1). After removing the duplicates, 358 papers were screened. Twenty-three full texts were verified. 16 were excluded and seven were included in the meta-analysis.

Table 1 reports the characteristics of the primary studies and results of outcomes. Table 2 shows the results of meta-analysis. We highlight that the papers included in this meta-analysis showed a low risk of bias: Supplementary Table S2 reports the results of risk bias assessment. The results of meta-regressions are showed in Table S3. Figures S1-S7 show results of sensitivity analysis.

Table 1. The characteristics of included studies, according to intervention group (curcumins) and control groups (placebo) and results for each selected outcome.

\begin{tabular}{|c|c|c|c|c|c|c|c|c|c|c|c|}
\hline $\begin{array}{l}\text { Author, } \\
\text { Year, } \\
\text { Country }\end{array}$ & $\begin{array}{l}\text { Mean } \\
\text { Age }\end{array}$ & $\underset{\%}{\text { Male }}$ & $\begin{array}{c}\text { Diabetes } \\
\text { Duration } \\
\text { (Years) }\end{array}$ & Groups & $\begin{array}{c}\text { BMI } \\
\text { Variation } \\
\text { Mean } \\
\text { (SD) }\end{array}$ & HOMA-IR & $\begin{array}{l}\text { Hb1Ac } \\
\text { Mean } \\
\text { (SD) }\end{array}$ & $\begin{array}{l}\text { TG } \\
\text { Mean } \\
\text { (SD) }\end{array}$ & $\begin{array}{c}\text { TC } \\
\text { Mean } \\
\text { (SD) }\end{array}$ & $\begin{array}{l}\text { HDL } \\
\text { Mean } \\
\text { (SD) }\end{array}$ & $\begin{array}{l}\text { LDL } \\
\text { Mean } \\
\text { (SD) }\end{array}$ \\
\hline \multirow{2}{*}{$\begin{array}{c}\text { Hodaei } \\
\text { 2019 Iran [10] }\end{array}$} & \multirow{2}{*}{59} & \multirow{2}{*}{50} & \multirow{2}{*}{$1-10$} & $\begin{array}{l}\text { Intervention } \\
\text { Group } \\
N=21 \\
(1500 \mathrm{mg}) \\
\end{array}$ & $\begin{array}{c}29.2 \\
(3.76) \\
28.9 \\
(3.73)\end{array}$ & $\begin{array}{c}62 \\
(63) \\
62.4 \\
(42) \\
\end{array}$ & $\begin{array}{l}11.3 \\
(1.6) \\
11 \\
(2.0) \\
\end{array}$ & - & - & - & - \\
\hline & & & & $\begin{array}{l}\text { Control } \\
\text { Group } \\
N=23\end{array}$ & $\begin{array}{l}28.2 \\
(2.5) \\
28.1 \\
(2.5)\end{array}$ & $\begin{array}{c}53 \\
(40) \\
65 \\
(44)\end{array}$ & $\begin{array}{l}11.2(1.3) \\
11.1(1.8)\end{array}$ & - & - & - & - \\
\hline \multirow{2}{*}{$\begin{array}{l}\text { Adibian } \\
2019 \\
\text { Iran [11] }\end{array}$} & \multirow{2}{*}{59} & \multirow{2}{*}{50} & \multirow{2}{*}{$1-10$} & $\begin{array}{l}\text { Intervention } \\
\text { Group } \\
N=21 \\
\text { (1500 mg) }\end{array}$ & - & - & - & $\begin{array}{l}124(36) \\
109(36)\end{array}$ & $\begin{array}{l}167(34) \\
163(39)\end{array}$ & $\begin{array}{l}30(2) \\
30(2)\end{array}$ & $\begin{array}{l}112(31) \\
108(36)\end{array}$ \\
\hline & & & & $\begin{array}{l}\text { Control } \\
\text { Group } \\
N=23\end{array}$ & - & - & - & $\begin{array}{l}126(52) \\
121(44)\end{array}$ & $\begin{array}{l}180(47) \\
175(47)\end{array}$ & $\begin{array}{l}30(2) \\
30(2)\end{array}$ & $\begin{array}{l}125(44) \\
118(47)\end{array}$ \\
\hline
\end{tabular}


Table 1. Cont.

\begin{tabular}{|c|c|c|c|c|c|c|c|c|c|c|c|}
\hline $\begin{array}{l}\text { Author, } \\
\text { Year, } \\
\text { Country }\end{array}$ & $\begin{array}{c}\text { Mean } \\
\text { Age }\end{array}$ & $\underset{\%}{\text { Male }}$ & $\begin{array}{c}\text { Diabetes } \\
\text { Duration } \\
\text { (Years) }\end{array}$ & Groups & $\begin{array}{c}\text { BMI } \\
\text { Variation } \\
\text { Mean } \\
\text { (SD) }\end{array}$ & HOMA-IR & $\begin{array}{l}\text { Hb1Ac } \\
\text { Mean } \\
\text { (SD) }\end{array}$ & $\begin{array}{l}\text { TG } \\
\text { Mean } \\
\text { (SD) }\end{array}$ & $\begin{array}{c}\text { TC } \\
\text { Mean } \\
\text { (SD) }\end{array}$ & $\begin{array}{l}\text { HDL } \\
\text { Mean } \\
\text { (SD) }\end{array}$ & $\begin{array}{c}\text { LDL } \\
\text { Mean } \\
\text { (SD) }\end{array}$ \\
\hline \multirow{2}{*}{ Adab 2018 Iran [12] } & \multirow{2}{*}{55} & \multirow{2}{*}{51} & \multirow{2}{*}{$5-10$} & $\begin{array}{l}\text { Intervention } \\
\text { Group } \\
N=39 \\
(2100 \mathrm{mg})\end{array}$ & $\begin{array}{l}28.98 \\
(3.68) \\
28.26 . \\
(3.45)\end{array}$ & $\begin{array}{c}2.42 \\
(1.73) \\
2.21 \\
(1.43)\end{array}$ & $\begin{array}{c}7.06 \\
(1.01) \\
7.04(0.98)\end{array}$ & $\begin{array}{l}181.56 \\
(79.9) \\
141.74 \\
(52.02)\end{array}$ & $\begin{array}{l}148.85 \\
(36.11) \\
149.82 \\
(35.67)\end{array}$ & $\begin{array}{c}38.79 \\
(10.30) \\
37.07 \\
(9.12)\end{array}$ & $\begin{array}{c}82.56 \\
(20.99) \\
75.23 \\
(18.84)\end{array}$ \\
\hline & & & & $\begin{array}{l}\text { Control } \\
\text { Group } \\
N=36\end{array}$ & $\begin{array}{l}28.82 \\
(4.96) \\
28.68 \\
(4.86)\end{array}$ & $\begin{array}{c}2.24 \\
(1.48) \\
2.69 \\
(2.02)\end{array}$ & $\begin{array}{c}6.79 \\
(1.08) \\
7.28(1.59)\end{array}$ & $\begin{array}{l}164.05 \\
(81.19) \\
197.05 \\
(96.98)\end{array}$ & $\begin{array}{l}155.36 \\
(36.27) \\
176.88 \\
(37.58)\end{array}$ & $\begin{array}{l}44.63 \\
(10.66) \\
42.11 \\
(9.39)\end{array}$ & $\begin{array}{c}86.61 \\
(21.99) \\
89.05 \\
(21.46)\end{array}$ \\
\hline \multirow[b]{2}{*}{$\begin{array}{c}\text { Rahimi } \\
2015 \\
\text { Iran [13] }\end{array}$} & \multirow[b]{2}{*}{58.64} & \multirow[b]{2}{*}{45} & \multirow[b]{2}{*}{ NR } & $\begin{array}{l}\text { Intervention } \\
\text { Group } \\
(80 \mathrm{mg}) \\
N=35\end{array}$ & $\begin{array}{l}26.92 \\
(2.71) \\
25.57 \\
(2.71)\end{array}$ & - & $\begin{array}{l}7.59 \text { (1.74) } \\
7.31(1.54)\end{array}$ & $\begin{array}{c}109 \\
(94.75) \\
131 \\
(60.27)\end{array}$ & $\begin{array}{l}163.4 \\
(33.94) \\
158.62 \\
(44.06)\end{array}$ & $\begin{array}{c}54.30 \\
(14.02) \\
60.95 \\
(15.68)\end{array}$ & $\begin{array}{c}96.57 \\
(33.94) \\
91.04 \\
(28.72)\end{array}$ \\
\hline & & & & $\begin{array}{l}\text { Control } \\
\text { Group } \\
N=35\end{array}$ & $\begin{array}{l}27.27 \\
(3.59) \\
27.50 \\
(3.38)\end{array}$ & - & $\begin{array}{l}7.49(1.75) \\
9.05(2.33)\end{array}$ & $\begin{array}{c}142(97.5) \\
113(58)\end{array}$ & $\begin{array}{c}85.5 \\
(15.3) \\
80.5 \\
(9.1)\end{array}$ & $\begin{array}{c}60.35 \\
(15.96) \\
55.00 \\
(11.09)\end{array}$ & $\begin{array}{c}98.78 \\
(30.33) \\
99.78 \\
(30.33) \\
84.00 \\
(12.59)\end{array}$ \\
\hline \multirow{2}{*}{$\begin{array}{c}\text { Chuengsamarn } \\
\text { Thailand } \\
2014 \text { [14] }\end{array}$} & \multirow{2}{*}{59} & \multirow{2}{*}{48} & \multirow{2}{*}{12} & $\begin{array}{l}\text { Intervention } \\
\text { Group } \\
N=107\end{array}$ & - & $\begin{array}{c}9.58 \\
(4.3) \\
4,32(1.8)\end{array}$ & - & $\begin{array}{l}219.12 \\
(97.52) \\
141.99 \\
(67.79) \\
\end{array}$ & - & - & - \\
\hline & & & & $\begin{array}{l}\text { Control } \\
\text { Groups } \\
N=106\end{array}$ & - & $\begin{array}{l}6.89(2.67) \\
6.78(2.5)\end{array}$ & - & $\begin{array}{c}252.72 \\
(114.12) \\
252 \\
(114.12)\end{array}$ & - & - & - \\
\hline \multirow{2}{*}{$\begin{array}{c}\mathrm{Na} \\
\text { China } \\
2013[15]\end{array}$} & \multirow{2}{*}{55.07} & \multirow{2}{*}{49} & \multirow{2}{*}{7.6} & $\begin{array}{l}\text { Intervention } \\
\text { Group } \\
(300 \mathrm{Mg}) \\
N=50\end{array}$ & - & $\begin{array}{c}5.80 \\
(3.35) \\
4.14(1.81)\end{array}$ & $\begin{array}{l}7.77(1.82) \\
7.02(2.04)\end{array}$ & $\begin{array}{l}223.8 \\
(46.8) \\
157.5 \\
(49.5) \\
\end{array}$ & $\begin{array}{c}540 \\
(100) \\
493 \\
(41.7) \\
\end{array}$ & $\begin{array}{c}52.9 \\
(10.04) \\
54.8 \\
(11.2) \\
\end{array}$ & $\begin{array}{l}166.0 \\
(46.3) \\
146.7 \\
(39.7) \\
\end{array}$ \\
\hline & & & & $\begin{array}{l}\text { Control } \\
\text { Group } \\
\mathrm{N}=50\end{array}$ & - & $\begin{array}{c}5.82 \\
(3.90) \\
5.49 \\
(2.15)\end{array}$ & $\begin{array}{l}7.72(2.12) \\
7.99(2.86)\end{array}$ & $\begin{array}{l}193.8 \\
(92.04) \\
186.7 \\
(66.3) \\
\end{array}$ & $\begin{array}{c}538 \\
(109) \\
522 \\
(105.3)\end{array}$ & $\begin{array}{c}51.35 \\
(10.81) \\
51.74 \\
(8.8)\end{array}$ & $\begin{array}{c}166.8 \\
(44.4) \\
160.2 \\
(45.17)\end{array}$ \\
\hline \multirow{2}{*}{$\begin{array}{l}\text { Usharani } \\
\text { India } \\
2008 \text { [16] }\end{array}$} & & \multirow{2}{*}{52} & \multirow{2}{*}{8} & $\begin{array}{l}\text { Intervention } \\
\text { Group } \\
N=23 \\
(300 \mathrm{mg})\end{array}$ & - & - & $\begin{array}{c}8.04 \\
(0.85) \\
8.03(0.76)\end{array}$ & $\begin{array}{l}176.39 \\
(27.61) \\
165.26 \\
(25.78)\end{array}$ & $\begin{array}{l}195.0 \\
(41.16) \\
185.34 \\
(34.35)\end{array}$ & $\begin{array}{l}38.78 \\
(7.69) \\
39.91 \\
(0.68)\end{array}$ & $\begin{array}{l}120.35 \\
(42.13) \\
111.34 \\
(37.65)\end{array}$ \\
\hline & & & & $\begin{array}{l}\text { Control } \\
\text { Group } \\
\mathrm{N}=21\end{array}$ & - & - & $\begin{array}{l}7.82(0.57) \\
7.80(0.62)\end{array}$ & $\begin{array}{l}170.14 \\
(47.54) \\
168.14 \\
(47.10)\end{array}$ & $\begin{array}{l}195.95 \\
(35.72) \\
198.76 \\
(35.09)\end{array}$ & $\begin{array}{l}36.38 \\
(7.67) \\
37.04 \\
(5.92)\end{array}$ & $\begin{array}{l}124.59 \\
(34.94) \\
122.18 \\
(35.56)\end{array}$ \\
\hline
\end{tabular}

\%: Males enrolled in each study.TG: triglycerides, TC: total cholesterol.

Table 2. Meta-Analysis results and moderator analysis (country: Iran versus outside Iran).

\begin{tabular}{|c|c|c|c|c|c|c|c|c|c|c|c|c|}
\hline \multirow[t]{2}{*}{ Outcome } & \multirow[t]{2}{*}{ K } & \multirow{2}{*}{$\begin{array}{c}\text { Total } \\
\text { Sample } \\
\text { Size }\end{array}$} & \multicolumn{2}{|l|}{ Effect Size } & \multicolumn{4}{|c|}{ Heterogeneity } & \multicolumn{4}{|c|}{ Publication Bias } \\
\hline & & & $(95 \% \mathrm{CI})$ & $p$ & $\mathrm{I}^{2}$ & $p$ & $\mathbf{T}^{2}$ & $\mathrm{~T}$ & $\begin{array}{c}\text { Egger } \\
(p)\end{array}$ & $\begin{array}{c}\text { BEGGS } \\
(p)\end{array}$ & $\begin{array}{c}\text { Fail Safe } \\
(n)\end{array}$ & $\begin{array}{l}\text { Rosenthal } \\
(n)\end{array}$ \\
\hline BMI & 3 & 168 & $-0.30(-0.62,0.02)$ & 0.067 & 0.00 & 0.514 & 0.00 & 0.00 & 0.842 & 0.602 & 0 & 25 \\
\hline $\mathrm{Hb} 1 \mathrm{Ac}$ & 5 & 333 & $-0.42(-0.77,-0.11)$ & 0.008 & 42.42 & 0.107 & 0.06 & 0.24 & 0.501 & 0.327 & 12 & 35 \\
\hline Iran & 3 & 189 & $-0.52(-1.00,-0.04)$ & 0.032 & 61.35 & 0.075 & 0.11 & 0.33 & & & & \\
\hline \multirow[t]{2}{*}{$\begin{array}{l}\text { Outside } \\
\text { Iran }\end{array}$} & 2 & 144 & $-0.28(-0.67,0.10)$ & 0.153 & 22.22 & 0.257 & 0.02 & 0.14 & & & & \\
\hline & \multicolumn{12}{|c|}{ ANOVA Q TEST $p=0.443$} \\
\hline HOMA & 4 & 432 & $-0.41(-0.66,-0.22)$ & $<0.001$ & 0.00 & 0.916 & 0.00 & 0.00 & 0.073 & 0.042 & 12 & 30 \\
\hline Iran & 2 & 119 & $-0.33(-0.69,0.04)$ & 0.078 & 0.00 & 0.667 & 0.00 & 0.00 & & & & \\
\hline $\begin{array}{l}\text { Outside } \\
\text { Iran }\end{array}$ & 2 & 313 & $-0.45(-0.67,-0.22)$ & $<0.001$ & 0.00 & 0.885 & 0.00 & 0.00 & & & & \\
\hline
\end{tabular}


Table 2. Cont.

\begin{tabular}{|c|c|c|c|c|c|c|c|c|c|c|c|c|}
\hline \multirow[t]{2}{*}{ Outcome } & \multirow[t]{2}{*}{$\mathbf{K}$} & \multirow{2}{*}{$\begin{array}{c}\text { Total } \\
\text { Sample } \\
\text { Size }\end{array}$} & \multicolumn{2}{|l|}{ Effect Size } & \multicolumn{4}{|c|}{ Heterogeneity } & \multicolumn{4}{|c|}{ Publication Bias } \\
\hline & & & $(95 \% \mathrm{CI})$ & $p$ & $I^{2}$ & $p$ & $\mathrm{~T}^{2}$ & $\mathbf{T}$ & $\underset{(p)}{\text { Egger }}$ & $\begin{array}{l}\text { BEGGS } \\
\quad(p)\end{array}$ & $\begin{array}{l}\text { Fail Safe } \\
\qquad(n)\end{array}$ & $\begin{array}{l}\text { Rosenthal } \\
\quad(n)\end{array}$ \\
\hline & \multicolumn{12}{|c|}{ ANOVA Q TEST $p=0.580$} \\
\hline HDL & 5 & 333 & $0.22(-0.08,0.52)$ & 0.143 & 45.91 & 0.116 & 0.05 & 0.27 & 0.856 & 0.327 & 1 & 35 \\
\hline Iran & 3 & 189 & $0.31(-0.21,0.839$ & 0.241 & 68.28 & 0.043 & 0.22 & 0.41 & & & & \\
\hline \multirow[t]{2}{*}{$\begin{array}{l}\text { Outside } \\
\text { Iran }\end{array}$} & 2 & 145 & $0.11(-0.22,0.43)$ & 0.527 & 0.00 & 0.713 & 0.00 & 0.00 & & & & \\
\hline & \multicolumn{12}{|c|}{ ANOVA Q TEST: $p=0.512$} \\
\hline LDL & 5 & 300 & $-0.28(-0.52,-0.04)$ & 0.021 & 0.00 & 0.083 & 0.00 & 0.00 & 0.646 & 0.624 & 1 & 35 \\
\hline Iran & 3 & 156 & $-0.32(-0.67,0.03)$ & 0.077 & 0.00 & 0.582 & 0.00 & 0.00 & & & & \\
\hline \multirow[t]{2}{*}{$\begin{array}{l}\text { Outside } \\
\text { Iran }\end{array}$} & 2 & 144 & $-0.25(-0.77,0.42)$ & 0.130 & 0.00 & 0.754 & 0.00 & 0.00 & & & & \\
\hline & \multicolumn{12}{|c|}{ ANOVA Q TEST: $p=0.793$} \\
\hline TG & 5 & 476 & $-0.57(-0.83,-0.31)$ & $<0.001$ & 41.56 & 0.144 & 0.04 & 0.19 & 0.943 & 0.322 & 37 & 35 \\
\hline Iran & 2 & 119 & $-0.59(-1.22,0.03)$ & 0.063 & 63.77 & 0.099 & 0.13 & 0.36 & & & & \\
\hline \multirow[t]{2}{*}{$\begin{array}{l}\text { Outside } \\
\text { Iran }\end{array}$} & 3 & 357 & $-0.55(-0.88,-0.22)$ & $<0.001$ & 49.19 & 0.140 & 0.04 & 0.20 & & & & \\
\hline & \multicolumn{12}{|c|}{ ANOVA Q TEST $p=0.904$} \\
\hline $\mathrm{TC}$ & 5 & 312 & $-0.30(-0.53,-0.07)$ & 0.01 & 0.00 & 0.573 & 0.00 & 0.00 & 0.975 & 1.00 & 3 & 30 \\
\hline Iran & 3 & 168 & $-0.27(-0.69,0.15)$ & 0.211 & 30.47 & 0.237 & 0.10 & 0.31 & & & & \\
\hline \multirow[t]{2}{*}{$\begin{array}{l}\text { Outside } \\
\text { Iran }\end{array}$} & 2 & 144 & $-0.32(-0.65,0.01)$ & 0.056 & 0.00 & 0.979 & 0.00 & 0.00 & & & & \\
\hline & \multicolumn{12}{|c|}{ ANOVA Q TEST $p=0.847$} \\
\hline
\end{tabular}

\subsection{BMI}

BMI was investigated in three studies $[10,12,13]$ involving a total of 168 patients. Overall, curcumin treatment does not show a statistically significant reduction between the treated and untreated patients: this results in the absence of statistical heterogeneity (Table 2, Figure 2).

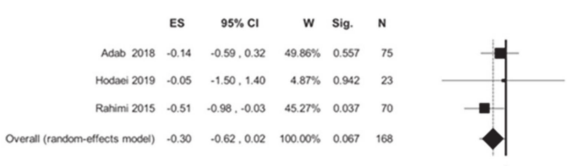

(a)

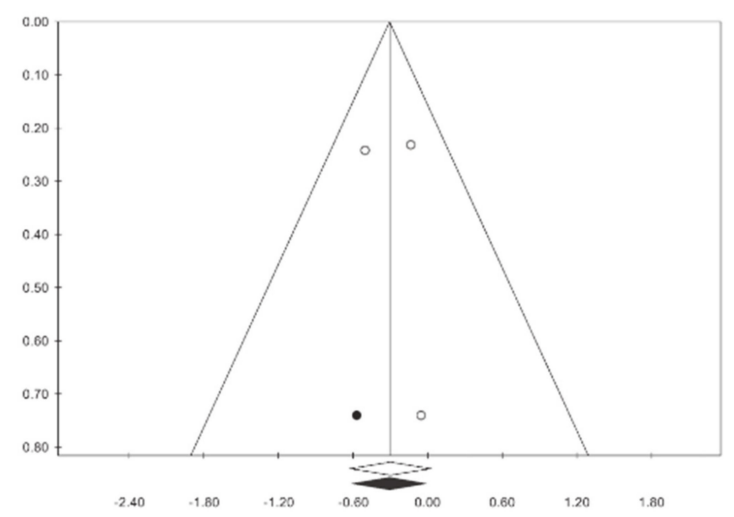

(b)

Figure 2. Meta-analysis results according to body mass index (BMI): (a) forest plot (b) funnel plot. 


\section{2. $H b 1 A c$}

Glycosylated hemoglobin was evaluated in five studies $[10,12,13,15,16]$. A statistically significant reduction was found in subjects that were treated with curcumin: $-0.42(-0.77$; $-0.11) p=0.008$, with moderate heterogeneity $\left(\mathrm{I}^{2}=42.42\right)$, but not statistically significant $(p=0.107)$. Publication bias analysis did not highlight any differences between the observed and estimated values. It should be emphasized that there is a difference between the studies conducted in Iran and those conducted in other countries (Table 2, Figure 3); however, this difference is not statistically significant (ANOVA $Q$ test $p=0.443$ ). The meta-regressions only showed statistical significance for gender $(p=0.034)$.

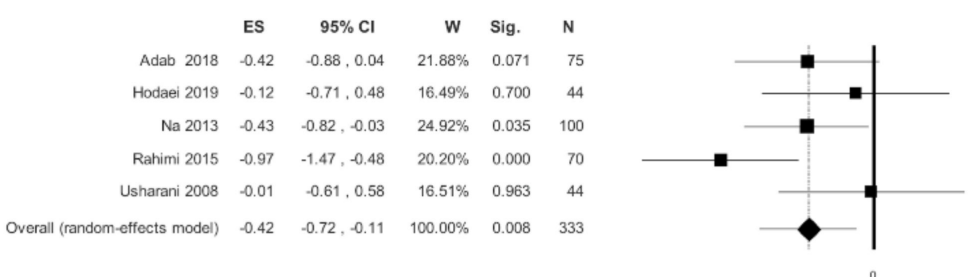

(a)

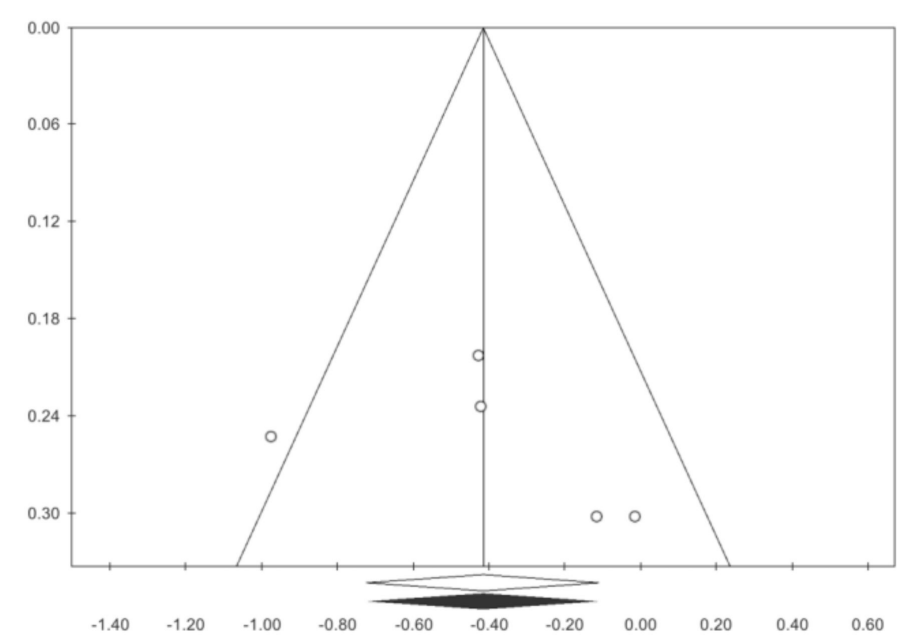

(b)

Figure 3. Meta-analysis results according to glycosylated hemoglobin (Hb1Ac): (a) forest plot (b) funnel plot.

\subsection{HOMA}

HOMA was detected in four studies $[10,12,14,15]$ for a total of 432 patients. There is a statistically significant reduction of this index, without statistical heterogeneity: -0.42 $(-0.77 ;-0.11) p<0.001$, (Table 2, Figure 4). Publication bias analysis highlighted a difference between the observed and estimated values: $0.45(-0.61 ;-0.28) p<0.001$, with two trimmed studies. The subgroup analysis showed a difference between the studies that were conducted in Iran and those conducted in other countries, however this difference is not statistically significant (Table 2). The meta-regressions, concerning the selected moderators, did not show any statistical significance (Table S3). 


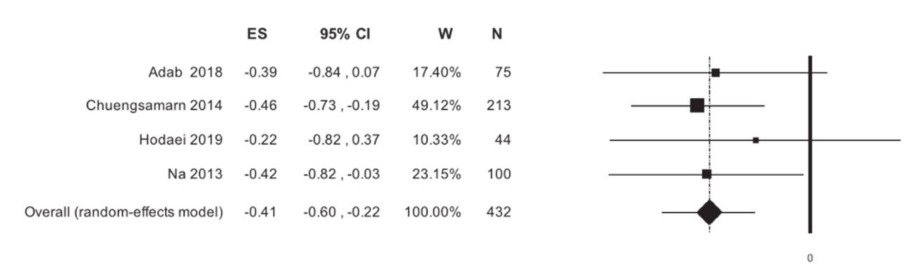

(a)

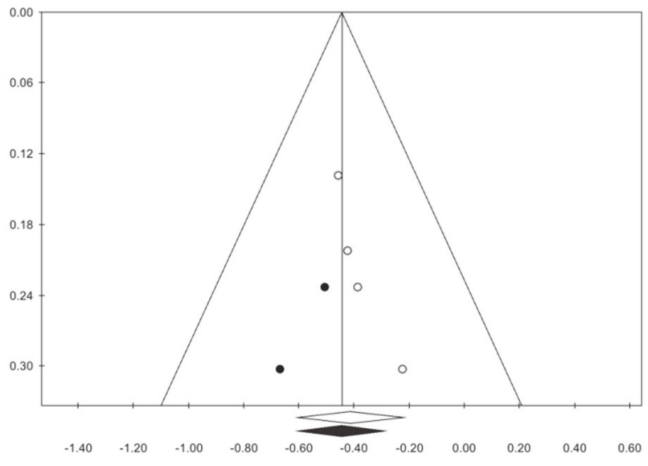

(b)

Figure 4. Meta-analysis results according to homeostasis model assessment (HOMA): (a) forest plot (b) funnel plot.

\subsection{HDL}

HDL was evaluated in five studies for a total of 333 patients $[11-13,15,16]$. The analysis did not show statistically significant differences (Table 2, Figure 5). The subgroup analysis did not show a difference between the studies that were conducted in Iran and those conducted in other countries. Publication bias analysis did not highlight any differences between the observed and estimated values. Meta-regressions, regarding the selected moderators, did not show any statistical significance, with the exception of gender $(p=0.002)$ (Table S3).

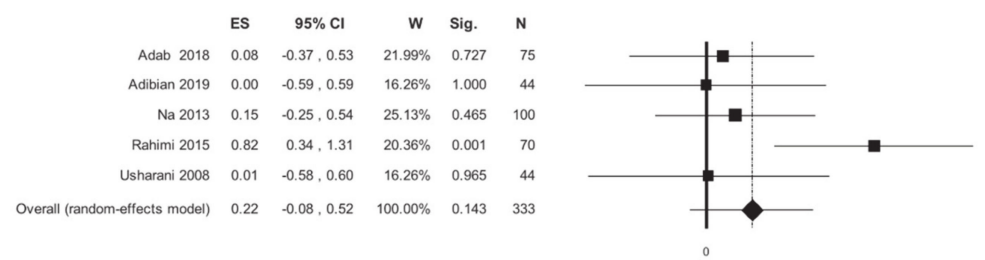

(a)

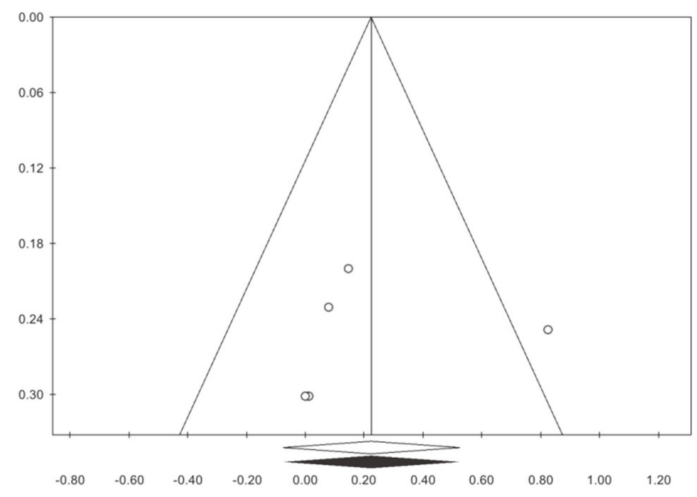

(b)

Figure 5. Meta-analysis results according to high-density lipoprotein (HDL): (a) forest plot (b) funnel plot. 


\section{5. $L D L$}

The LDL dosage was evaluated in 300 patients for a total of five studies [11-13,15,16]. The meta-analysis showed a statistically significant reduction in curcumin-treated patients when compared to the placebo, with no statistical heterogeneity: $-0.28(-0.52 ;-0.04)$ $p=0.021, \mathrm{I}^{2} 0.00$ (Table 2, Figure 6). The subgroup analysis, as shown in Table 2, highlights a difference between studies that were conducted in Iran and those conducted outside Iran, but there is not a statistically significant difference. Publication bias analysis did not highlight any differences between the observed and estimated values. The meta-regressions, for the selected moderators, did not show any statistical significance (Table S3).

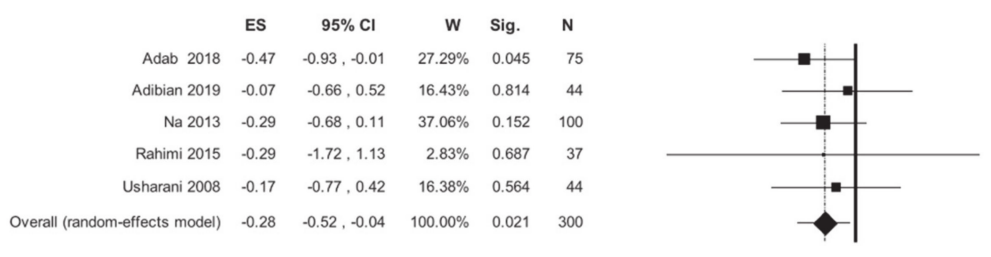

(a)

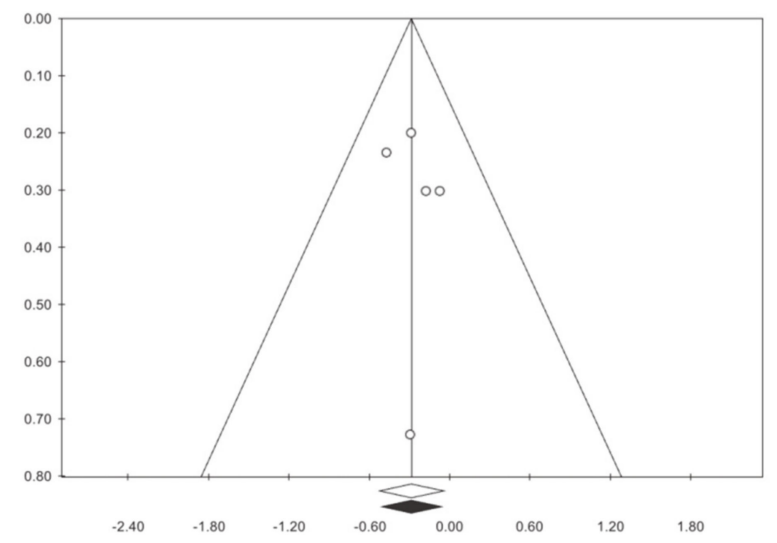

(b)

Figure 6. Meta-analysis results according to low-density lipoprotein (LDL): (a) forest plot (b) funnel plot.

\subsection{Triglycerides}

Triglycerides were evaluated in five primary studies, involving a total of 476 patients [11-16]. In patients treated with curcumin, a non-significant reduction in plasma triglyceride concentrations, without statistical heterogeneity, was identified (Table 2, Figure 7). The publication bias analysis highlighted a difference between observed and estimated values, respectively: $-0.62(-0.87 ;-0.37)(p<0.001),-0.57(-0.83 ;-0.31)$, with 1 trimmed study. The subgroup analysis revealed a non-statistically significant difference.

\subsection{Total Cholesterol}

The total cholesterol was investigated in five studies for a total of 312 statistical units $[11-13,15,16]$. There is a reduction in cholesterol in curcumin-treated patients as compared to the placebo-treated patients, with no statistical heterogeneity (Table 2, Figure 8). Publication bias analysis highlighted a difference between the observed and estimated values, respectively: $-0.30(-0.53 ;-0.07)$ $p<0.001,-0.40(-0.62 ;-0.28)(p<0.001)$ with two trimmed studies. No statistically significant associations were found in meta-regressions (Table S3). 


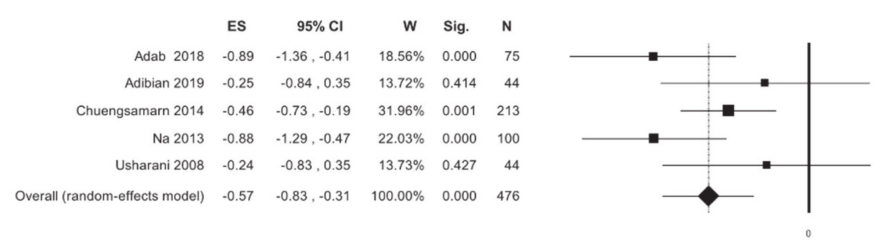

(a)

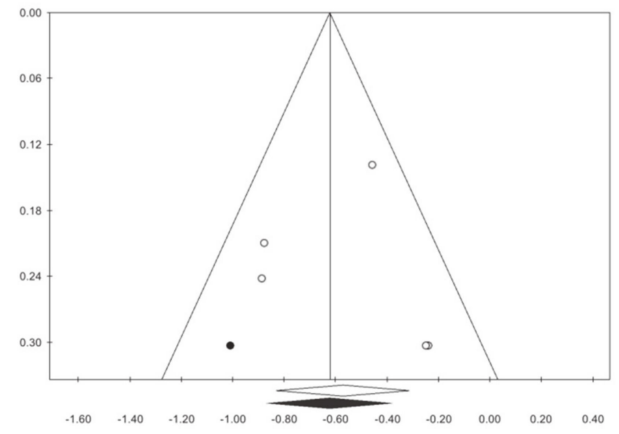

(b)

Figure 7. Meta-analysis results according to Triglycerides: (a) forest plot (b) funnel plot.

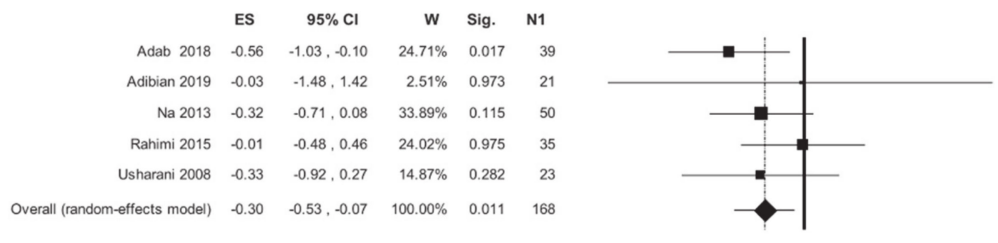

(a)

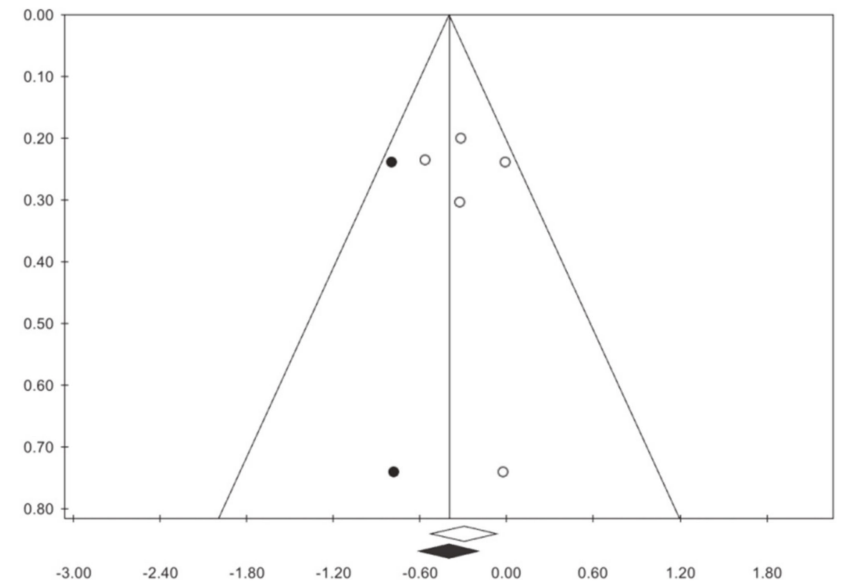

(b)

Figure 8. Meta-analysis results according to Total Cholesterol: (a) forest plot (b) funnel plot.

\section{Discussion}

In recent decades, there has been considerable interest among researchers in nutraceuticals and in particular, in naturally derived products, also known as natural health products (NHP), for the prevention, cure, and treatment of cardiovascular and metabolic diseases [24-26]. Some research indicates that chronically ill people tend to consume more NHP, and some surveys confirm that patients with T2DM are not excluded from this [27-31]. The pandemic spread of non-communicable diseases (NCD), and T2DM in particular, makes this 
population a target market of considerable interest for producers. The use of NHP in diabetics is linked to the co-treatment of hyperglycemia, dyslipidemia, and complications of diabetes. Among the various products, there is also curcumin. Currently, the FDA and EFSA recommend doses of curcumin of maximum $3 \mathrm{mg} / \mathrm{kg} / \mathrm{day}$, including the onset of toxicity for higher quantities (in particular, teratogenic effects, astrocytic cell abnormalities are reported, and it is not recommended in gallbladder stones) [5]. Overall, curcumin has a low bioavailability due to its hydrophobicity, so the pharmacologically active form is administered with a lipid vehicle or in association with piperine $[5,32]$. Specifically, for diabetes, curcumin also has an effect on hepatic lipogenesis, blocking the activity of the sterol regulatory element-binding protein gene (SREBP1) [6,33] and simultaneously activating the enzymes carnitine palmitoyltransferase 1 (CPT1) and acyl-CoA cholesterol acyltransferase (ACAT) that are involved in lipid mobilization [6,33].

The results of our meta-analysis seem to confirm this modulating capacity on lipid metabolism. The trials considered highlighted an overall reduction in LDL, TG, and TC in patients with uncomplicated T2DM. This result does not seem to be affected by statistical heterogeneity. There is a moderate publication bias. The low fail-safe for LDL, HDL, and TC indicates caution in the interpretation of the overall result, even if a high Rosenthal value allows for the observation found to be considered valid [22]. In order to confirm this, the subgroup analysis shows some differences that could be explained by the following considerations: method of conducting the study, quantity of curcumin administered, execution techniques, analysis, and collection of the blood chemistry method. The lack of influence of curcumin on HDL can be motivated by the fact that, notoriously, the increase in HDL is due globally to a more active lifestyle [34]. Similarly, the non-influence on BMI could be interpreted [35].

Because hypoglycemic properties of curcumin have been known since 1972 [36,37], the action is probably mediated by the inhibition of Phosporilase Kinase, which is to say, avoiding the mobilization of glucose from glycogen reserves [32,37-39]. Furthermore, curcumin would have a role in reducing the accumulation of advanced glycation end products [40] and the accumulation of these same metabolites at the level of the pancreatic insulae [41]. The inhibition of this process would mediate the Peroxisome Proliferator Activated Receptor Gamma (PPAR-Y), which, by increasing the amount of glutathione, would prevent oxidative damage that is caused by the state of hyperglycemia [42].

$\mathrm{Hb} 1 \mathrm{Ac}$ and HOMA show significant results without statistical heterogeneity and publication bias. Regarding $\mathrm{Hb} 1 \mathrm{Ac}$, the subgroup analysis shows a difference between the studies that were conducted in Iran and those conducted outside Iran; on the contrary, this is not the case for HOMA, which shows concordant results. The hypoglycemic capacity of curcumin has also been tested in prediabetic populations with some success [43].

Concerning $\mathrm{Hb} 1 \mathrm{Ac}$ and $\mathrm{HDL}$, the results of the meta-regressions only show statistical significance with respect to gender.

\section{Conclusions}

Our meta-analysis seems to confirm the benefits on glucose metabolism, with results that appear to be more solid than those of lipid metabolism. In conclusion, the daily supplement of curcumin could improve some metabolic aspects of uncomplicated T2DM patients.

However, further studies are needed in order to test the efficacy and safety of curcumin in uncomplicated T2DM. The limitations of the present work can be attributed to some biases present in the primary studies: the small numbers of enrolled patients and the possible impact of grey literature. These aspects cannot be totally corrected through the meta-analytic technique.

Supplementary Materials: The following are available online at https:/ / www.mdpi.com/2072-6 643/13/2/404/s1, Table S1: Prisma Checklist, Table S2: Risk of bias assessment, Table S3: Metaregressions Results, Figures S1-S7: Sensitivity Analysis. 
Author Contributions: E.A.: Guarantor of the article, study concept and design, literature search, data analysis, and manuscript writing. P.M.A.: literature search, data abstraction, participant manuscript writing. M.M., A.R.G. and C.M. participant systematic review (PRISMA). R.P. participant study concept and manuscript writing. All authors have read and agreed to the published version of the manuscript.

Funding: This research received no external funding.

Founding: The founding sponsors had no role in the design of the study; in the collection, analyses, or interpretation of data; in the writing of the manuscript, and in the decision to publish the results.

Data Availability Statement: Not applicable.

Conflicts of Interest: The authors declare no conflict of interest.

\section{References}

1. World Health Organization (WHO). World Health Statistics 2016: Monitoring Health for the SDGs Sustainable Development Goals; World Health Organization: Geneva, Switzerland, 2016.

2. Altobelli, E.; Angeletti, P.M.; Profeta, V.F.; Petrocelli, R. Lifestyle risk factors for type 2 diabetes mellitus and national diabetes care systems in European countries. Nutrients 2020, 12, 2806. [CrossRef] [PubMed]

3. Bommer, C.; Sagalova, V.; Heesemann, E.; Manne-Goehler, J.; Atun, R.; Bärnighausen, T.; Davies, J.; Vollmer, S. Global economic burden of diabetes in adults: Projections from 2015 to 2030. Diabetes Care 2018, 41, 963-970. [CrossRef] [PubMed]

4. Kim, H.S.; Hwang, Y.C.; Koo, S.H.; Park, K.S.; Lee, M.S.; Kim, K.W.; Lee, M.K. PPAR-gamma activation increases insulin secretion through the up-regulation of the free fatty acid receptor GPR40 in pancreatic beta-cells. PLoS ONE 2013, 8, e50128.

5. Shaikh, J.; Ankola, D.D.; Beniwal, V.; Singh, D.; Kumar, M.N. Nanoparticle encapsulation improves oral bioavailability of curcumin by at least 9-fold when compared to curcumin administered with piperine as absorption enhancer. Eur. J. Pharm. Sci. 2009, 37, 223-230. [CrossRef] [PubMed]

6. Pivari, F.; Mingione, A.; Brasacchio, C.; Soldati, L. Curcumin and type 2 diabetes mellitus: Prevention and treatment. Nutrients 2019, 11, 1837. [CrossRef]

7. Aggarwal, B.B.; Harikumar, K.B. Potential therapeutic effects of curcumin, the anti-inflammatory agent, against neurodegenerative, cardiovascular, pulmonary, metabolic, autoimmune and neoplastic diseases. Int. J. Biochem. Cell Biol. 2009, 41, 40-59. [CrossRef]

8. Ramírez-Boscá, A.; Soler, A.; Carrión, M.A.; Díaz-Alperi, J.; Bernd, A.; Quintanilla, C.; Quintanilla Almagro, E.; Miquel, J. An hydroalcoholic extract of curcuma longa lowers the apo B/apo A ratio. Implications for atherogenesis prevention. Mech. Ageing Dev. 2000, 20, 41-47.

9. Mohammadi, A.; Sahebkar, A.; Iranshahi, M.; Amini, M.; Khojasteh, R.; Ghayour-Mobarhan, M.; Ferns, G.A. Effects of supplementation with curcuminoids on dyslipidemia in obese patients: A randomized crossover trial. Phytother. Res. 2013, 27, 374-379. [CrossRef]

10. Hodaei, H.; Adibian, M.; Nikpayam, O.; Hedayati, M.; Sohrab, G. The effect of curcumin supplementation on anthropometric indices, insulin resistance and oxidative stress in patients with type 2 diabetes: A randomized, double-blind clinical trial. Diabetol. Metab. Syndr. 2019, 27, 41. [CrossRef]

11. Adibian, M.; Hodaei, H.; Nikpayam, O.; Sohrab, G.; Hekmatdoost, A.; Hedayati, M. The effects of curcumin supplementation on high-sensitivity C-reactive protein, serum adiponectin, and lipid profile in patients with type 2 diabetes: A randomized, double-blind, placebo-controlled trial. Phytother. Res. 2019, 33, 1374-1383. [CrossRef]

12. Adab, Z.; Eghtesadi, S.; Vafa, M.R.; Heydari, I.; Shojaii, A.; Haqqani, H.; Arablou, T.; Eghtesadi, M. Effect of turmeric on glycemic status, lipid profile, Hs-CRP, and total antioxidant capacity in hyperlipidemic type 2 diabetes mellitus patients. Phytother. Res. 2019, 33, 1173-1181. [CrossRef] [PubMed]

13. Rahimi, H.R.; Mohammadpour, A.H.; Dastani, M.; Jaafari, M.R.; Abnous, K.; Ghayour Mobarhan, M.; Kazemi Oskuee, R. The effect of nano-curcumin on $\mathrm{HbA1c}$, fasting blood glucose, and lipid profile in diabetic subjects: A randomized clinical trial. Avicenna J. Phytomed. 2016, 6, 567-577. [PubMed]

14. Chuengsamarn, S.; Rattanamongkolgul, S.; Phonrat, B.; Tungtrongchitr, R.; Jirawatnotai, S. Reduction of atherogenic risk in patients with type 2 diabetes by curcuminoid extract: A randomized controlled trial. J. Nutr. Biochem. 2014, 25, 144-150. [CrossRef] [PubMed]

15. Na, L.X.; Li, Y.; Pan, H.Z.; Zhou, X.L.; Sun, D.J.; Meng, M.; Li, X.X.; Sun, C.H. Curcuminoids exert glucose-lowering effect in type 2 diabetes by decreasing serum free fatty acids: A double-blind, placebo-controlled trial. Mol. Nutr. Food Res. 2013, 57, 1569-1577. [CrossRef] [PubMed]

16. Usharani, P.; Mateen, A.A.; Naidu, M.U.; Raju, Y.S.; Chandra, N. Effect of NCB-02, atorvastatin and placebo on endothelial function, oxidative stress and inflammatory markers in patients with type 2 diabetes mellitus: A randomized, parallel-group, placebo-controlled, 8-week study. Drugs Res. Dev. 2008, 9, 243-250. [CrossRef]

17. Moher, D.; Liberati, A.; Tetzlaff, J.; Altman, D.G. PRISMA Group. Preferred reporting items for systematic reviews and meta-analyses: The PRISMA statement. PLoS Med. 2009, 21, e1000097. 
18. Higgins, J.P.T.; Green, S.; Altman, D.G. Cochrane Handbook for Systematic Reviews of Interventions: Cochrane Book Series Chapter 8; Assessing Risk of Bias in Included Studies Published Online; Wiley and Sons: Hoboken, NJ, USA, 2008.

19. Higgins, J.P.T.; Thompson, S.G.; Spiegelhalter, D.J. A re-evaluation of random-effects meta-analysis. J. R. Stat. Soc. 2009, 172, 137-159. [CrossRef]

20. Rhea, M.R. Determining the magnitude of treatment effects in strength training research through the use of the effect size. J. Strength Cond. Res. 2004, 18, 918-920.

21. Rothstein, H.R.; Sutton, A.J.; Borenstein, M. Publication Bias in Meta-Analysis; Wiley and Sons: Chichester, UK, 2005.

22. Fragkos, K.C.; Tsagris, M.; Frangos, C.C. Publication bias in meta-analysis: Confidence intervals for Rosenthal's fail-safe number. Int. Sch. Res. Notices. 2014, 2014, 825383. [CrossRef]

23. Rugge, B.; Balshem, H.; Sehgal, R.; Relevo, R.; Gorman, P.; Helfand, M. Screening and Treatment of Subclinical Hypothyroidism or Hyperthyroidism; Comparative Effectiveness Reviews, No. 24; Agency for Healthcare Research and Quality: Rockville, MD, USA, 2011 Available online: https:/ / www.ncbi.nlm.nih.gov/books/NBK83496/ (accessed on 25 January 2021).

24. Waltenberger, B.; Mocan, A.; Šmejkal, K.; Heiss, E.H.; Atanasov, A.G. Natural products to counteract the epidemic of cardiovascular and metabolic disorders. Molecules 2016, 22, 807. [CrossRef]

25. Dwyer, J.T.; Coates, P.M.; Smith, M.J. Dietary supplements: Regulatory challenges and research resources. Nutrients 2018, 10, 41. [CrossRef] [PubMed]

26. Kantor, E.D.; Rehm, C.D.; Du, M.; White, E.; Giovannucci, E.L. Trends in dietary supplement use among US adults from $1999-2012$. JAMA Intern. Med. 2016, 316, 1464-1474. [CrossRef] [PubMed]

27. Geil, P.; Shane-McWhorter, L. Dietary supplements in the management of diabetes: Potential risks and benefits. J. Am. Diet. Assoc. 2008, 8, S59-S65. [CrossRef] [PubMed]

28. Alherbish, A.; Charrois, T.L.; Ackman, M.L.; Tsuyuki, R.T.; Ezekowitz, J.A. The prevalence of natural health product use in patients with acute cardiovascular disease. PLoS ONE 2011, 6, e19623. [CrossRef]

29. Necyk, C.; Zubach-Cassano, L. Natural health products and diabetes: A practical review. Can. J. Diabetes 2017, 41, 642-647. [CrossRef]

30. Damnjanovic, I.; Kitic, D.; Stefanovic, N.; Zlatkovic-Guberinic, S.; Catic-Djordjevic, A.; Velickovic-Radovanovic, R. Herbal self-medication use in patients with diabetes mellitus type 2. Turk. J. Med. Sci. 2015, 45, 964-971. [CrossRef]

31. Heller, T.; Müller, N.; Kloos, C.; Wolf, G.; Müller, U.A. Self-medication and use of dietary supplements in adult patients with endocrine and metabolic disorders. Exp. Clin. Endocrinol. Diabetes 2012, 120, 540-546. [CrossRef]

32. Kunnumakkara, A.B.; Bordoloi, D.; Padmavathi, G.; Monisha, J.; Roy, N.K.; Prasad, S.; Aggarwal, B.B. Curcumin, the golden nutraceutical: Multitargeting for multiple chronic diseases. Br. J. Pharmacol. 2017, 174, 1325-1348. [CrossRef]

33. Seo, K.I.; Choi, M.S.; Jung, U.J.; Kim, H.J.; Yeo, J.; Jeon, S.M.; Lee, M.K. Effect of curcumin supplementation on blood glucose, plasma insulin, and glucose homeostasis related enzyme activities in diabetic Db/Db mice. Mol. Nut. Food Res. 2008, 52, 995-1004. [CrossRef]

34. Escolà-Gil, J.C.; Julve, J.; Griffin, B.A.; Freeman, D.; Blanco-Vaca, F. HDL and lifestyle interventions. Handb. Exp. Pharmacol. 2015, 224, 569-592.

35. Lv, N.; Azar, K.M.J.; Rosas, L.G.; Wulfovich, S.; Xiao, L.; Ma, J. Behavioral lifestyle interventions for moderate and severe obesity: A systematic review. Prev. Med. 2017, 100, 180-193. [CrossRef] [PubMed]

36. Gupta, S.C.; Patchva, S.; Aggarwal, B.B. Therapeutic roles of curcumin: Lessons learned from clinical trials. AAPS J. 2013, 15, 195-218. [CrossRef] [PubMed]

37. Srinivasan, M. Effect of curcumin on blood sugar as seen in a diabetic subject. Indian J. Med. Sci. 1972, 26, 269-270. [PubMed]

38. Aggarwal, B.B.; Deb, L.; Prasad, S. Curcumin differs from tetrahydrocurcumin for molecular targets, signaling pathways and cellular responses. Molecules 2014, 20, 185-205. [CrossRef] [PubMed]

39. Reddy, S.; Aggarwal, B.B. Curcumin is a non-competitive and selective inhibitor of phosphorylase kinase. FEBS Lett. 1994, 341, 19-22. [CrossRef]

40. Sajithlal, G.B.; Chithra, P.; Chandrakasan, G. Advanced glycation end products induce crosslinking of collagen in vitro. Biochim. Biophys. Acta 1998, 1407, 215-224. [CrossRef]

41. Meghana, K.; Sanjeev, G.; Ramesh, B. Curcumin prevents streptozotocin-induced islet damage by scavenging free radicals: A prophylactic and protective role. Eur. J. Pharmacol. 2007, 577, 183-191. [CrossRef]

42. Stefanska, B. Curcumin ameliorates hepatic fibrosis in type 2 diabetes mellitus-Insights into its mechanisms of action. Br. J. Pharmacol. 2012, 166, 2209-2211. [CrossRef]

43. Lee, M.S.; Wahlqvist, M.L.; Chou, Y.C.; Fang, W.H.; Lee, J.T.; Kuan, J.C.; Liu, H.Y.; Lu, T.M.; Xiu, L.; Hsu, C.C.; et al. Turmeric improves post-prandial working memory in pre-diabetes independent of insulin. Asia Pac. J. Clin. Nutr. 2014, 23, 581-591. 